

\title{
Doseur continu d'hydrocarbures gazeux fonctionnant en méthode de zéro asservi
}

\author{
A. Jutard, S. Scavarda, M. Lebrun, M. Betemps
}

\section{To cite this version:}

A. Jutard, S. Scavarda, M. Lebrun, M. Betemps. Doseur continu d'hydrocarbures gazeux fonctionnant en méthode de zéro asservi. Revue de Physique Appliquée, 1975, 10 (5), pp.309-318. 10.1051/rphysap:01975001005030900 . jpa-00243918

\section{HAL Id: jpa-00243918 https://hal.science/jpa-00243918}

Submitted on 1 Jan 1975

HAL is a multi-disciplinary open access archive for the deposit and dissemination of scientific research documents, whether they are published or not. The documents may come from teaching and research institutions in France or abroad, or from public or private research centers.
L'archive ouverte pluridisciplinaire HAL, est destinée au dépôt et à la diffusion de documents scientifiques de niveau recherche, publiés ou non, émanant des établissements d'enseignement et de recherche français ou étrangers, des laboratoires publics ou privés. 


\title{
Classification \\ Physics Abstracts \\ 0.695 \\ DOSEUR CONTINU D'HYDROCARBURES GAZEUX FONCTIONNANT EN MÉTHODE DE ZÉRO ASSERVI
}

\author{
A. JUTARD, S. SCAVARDA, M. LEBRUN et M. BETEMPS \\ Laboratoire de « Mesure et Contrôle », Département de Génie Mécanique Construction \\ Institut National des Sciences Appliquées de Lyon \\ Bât. 303, 20, avenue A. Einstein, 69621 Villeurbanne, France
}

(Reçu le 3 février 1975, accepté le 21 avril 1975)

\begin{abstract}
Résumé. - Le dosage des hydrocarbures gazeux contenus dans un mélange air-combustible peut être obtenu en mesurant les effets du dégagement de chaleur dû à leur combustion catalytique sur filament de platine incandescent. Cet article présente une réalisation originale d'un analyseur continu de gaz combustible basé sur ce principe. L'utilisation d'une méthode de zéro asservi permet de pallier les défauts rencontrés dans ce type d'appareil : durée de vie des filaments, dérive de zéro, temps de réponse élevé.
\end{abstract}

\begin{abstract}
The continuous determination of small quantities of hydrocarbons in gaseous mixtures can be performed by measuring the heat emission due to catalytic combustion on an incandescent platinum filament. The present paper describes a novel continuous hydrocarbon analyser based upon this principle. The use of automatic zero control results in an improved performance with regard to : life of filaments, zero shift and shorter response time.
\end{abstract}

1. Méthode de dosage des hydrocarbures. - Le problème de la détection et du dosage d'un constituant combustible dans un mélange gazeux, et particulièrement dans l'air, intéresse de nombreux secteurs de l'industrie : détection des hydrocarbures, analyse de l'atmosphère dans les mines et certains laboratoires [1], vérification de l'étanchéité : des vannes, réservoirs et conduites dans les raffineries [2], pollution atmosphérique $[3], \ldots$

Différentes techniques sont envisageables pour réaliser cette détection ; pour les hydrocarbures gazeux, ces méthodes sont dites chimiques ou physiques suivant que la molécule est détruite ou non par le dosage [1].

Ainsi, il existe de nombreux appareils basés sur la mesure d'une propriété physique de l'hydrocarbure considéré : densité, indice de réfraction $[4,5]$, conductibilité thermique $[6,7]$, absorption du rayonnement infrarouge [8].

Les méthodes dites chimiques mettent toutes en œuvre l'oxydation des hydrocarbures ; pour le méthane par exemple, on obtient la réaction finale :

$$
\mathrm{CH}_{4}+2 \mathrm{O}_{2} \rightarrow \mathrm{CO}_{2}+2 \mathrm{H}_{2} \mathrm{O} \text {. }
$$

Cette réaction s'accompagne d'une variation d'enthalpie standard [9]:

$$
\Delta \mathrm{H}_{298}^{0}=-191,7 \mathrm{~K} \cdot \mathrm{cal} \cdot \mathrm{mole}^{-1}
$$

et d'une émission U. V.
La présence d'un catalyseur, constitué d'un filament de platine porté électriquement à l'incandescence, favorise la réaction exothermique (1).

Cette combustion catalytique peut être mise en évidence de différentes façons comme l'indique A. Monomakhoff [1] : par l'auto-entretien de la réaction, par la mesure de la diminution du volume des gaz brûlés, par l'évaluation des quantités de vapeur d'eau et de gaz carbonique formées, par la détection de l'absorption U. V. ou par la mesure du dégagement de chaleur ou de ses effets.

En particulier, le dégagement de chaleur dû à la réaction provoque une élévation des températures du mélange et du filament, d'où une variation de la résistance du filament.

Un certain nombre d'appareils réalisent la mesure de la teneur en hydrocarbure du mélange en exploitant les effets secondaires de la combustion catalytique sur la résistance du filament de mesure.

Les doseurs de méthane du type Leon-Montluçon ou du type M. S. A. [10] admettent le mélange à analyser d'une façon discontinue dans la chambre de combustion contenant le filament de mesure. Un autre filament, servant de référence, baigne dans un volume fermé d'air pur. Ces deux filaments constituent deux bras d'un pont de Wheatstone dont le déséquilibre, au moment de la combustion, est fonction de la quantité de combustible brûlé. On convient de prendre 
comme indication de la teneur le déséquilibre maximal observé entre le début et la fin de la réaction.

Or, il est bien connu [11] que l'on observe une augmentation de l'activité catalytique du filament dans le temps qui se traduit par une réaction plus rapide et donc par une élévation plus importante de la température du filament.

De ce fait, ces appareils présentent différents inconvénients :

- la mesure, consistant à prendre au vol l'indication maximale de l'appareil de zéro, est affectée par cette variation d'activité, d'où une variation non négligeable de l'étalonnage,

- lorsque le filament devient très actif, les élévations brutales de sa température favorisent sa destruction rapide,

- la variation lente de la résistance du filament de mesure due à l'évaporation de platine introduit une dérive du zéro entraînant la nécessité d'un équilibrage avant chaque mesure,

- le fonctionnement de l'appareil est discontinu.

Signalons que des appareils découlant de ce principe et mis au point par le CERCHAR diminuent considérablement ces défauts, sans toutefois les éliminer totalement $[12,13,14]$.

Les effets de la variation d'activité du filament peuvent être rendus négligeables en balayant celui-ci par le mélange à analyser à un débit suffisamment faible pour que les gaz résiduels soient quasiment épuisés en méthane [15]. Il s'établit alors un régime continu de combustion et la durée de vie du filament s'en trouve accrue.

En vue de limiter la dérive de zéro de ces doseurs continus, un principe original [16] consiste à remplacer la mesure différentielle dans l'espace, effectuée sur deux filaments distincts, par une mesure différentielle dans le temps sur un filament unique, les déterminations alternées en combustion et en référence étant réalisées à une fréquence telle qu'un appareil intégrateur en fournira une indication continue.

Il est possible aussi d'évaluer la teneur en hydrocarbure du mélange en mesurant la température du gaz qui entoure le filament où a lieu la réaction.

Nous avons antérieurement conçu un analyseur continu d'hydrocarbures gazeux dans l'air s'appuyant sur ce principe [17], [18]. L'élément catalytique, constitué par un filament de platine incandescent, est placé dans l'une des deux cellules d'un calorimètre différentiel à flux de chaleur intégral, l'autre cellule recevant un filament de référence. Le mélange gazeux, circulant à travers les deux cellules à vitesse réduite, subit une combustion totale dans la cellule de mesure. La thermopile, placée entre les deux cellules, fournit une force électromotrice directement proportionnelle au flux de chaleur entre les deux cellules du calorimètre, le flux de chaleur étant lui-même proportionnel à la teneur en gaz combustible.

Cet analyseur, s'il élimine les influences des varia- tions d'activité du catalyseur, présente quand même une dérive du zéro due au vieillissement du filament. D'autre part, les capteurs thermoélectriques étant placés à l'extérieur des cellules de combustion et de référence, le temps de réponse de l'appareil est assez long.

En fait, on peut noter une certaine analogie entre cet appareil et un débitmètre thermique, au rôle près des éléments chauffants. Cette remarque nous a conduit à concevoir un doseur plus simple, très proche d'un débitmètre thermique.

Nous présentons dans cet article :

- les solutions adoptées pour la réalisation d'un doseur simplifié palliant, dans la mesure du possible, les effets des différents défauts signalés ci-dessus;

- les améliorations supplémentaires dues à un fonctionnement suivant une méthode de zéro asservi.

2. Etude théorique et expérimentale d'un analyseur. - 2.1 DesCription DE L'ANALYSEUR. - Le mélange à analyser s'écoule dans le tube à débit constant ; il rencontre un premier filament de platine, ou filament de mesure, porté à une température suffisante pour provoquer la combustion catalytique de l'hydrocarbure contenu dans le mélange. On choisit un débit tel qu'il y ait combustion complète sur ce filament. Un deuxième filament sert de référence. Les couples thermoélectriques, constituant la thermopile, sont situés au sein du fluide en aval des filaments (Fig. 1). La température du second filament est réglée de façon à annuler la tension délivrée par la thermopile en l'absence d'hydrocarbures dans le courant gazeux. La tension de sortie de la thermopile est directement liée à la quantité d'hydrocarbure brûlé sur le filament de mesure.

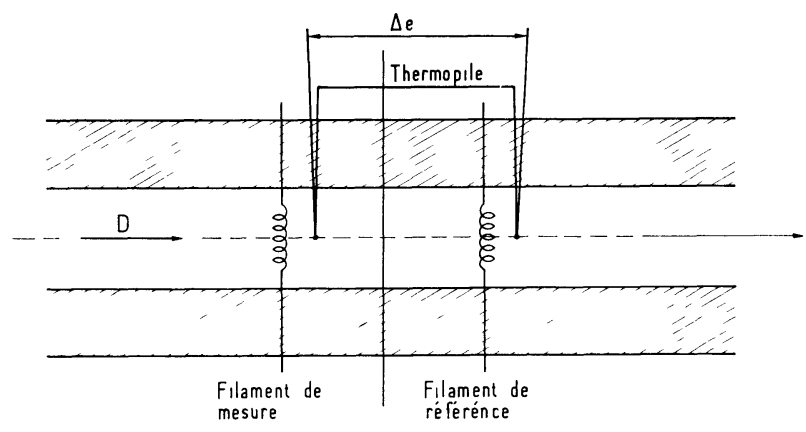

FIG. 1. - Schéma de principe du doseur.

On admet généralement [19] que la combustion se produit à la surface du platine et qu'elle peut se poursuivre dans son voisinage immédiat. Il est donc légitime de supposer que l'apport de chaleur $Q$ dû à la réaction est uniquement réparti au niveau du premier filament.

La forme et la disposition des différents éléments constituant le doseur apparaissent sur le dessin de la figure 2. 


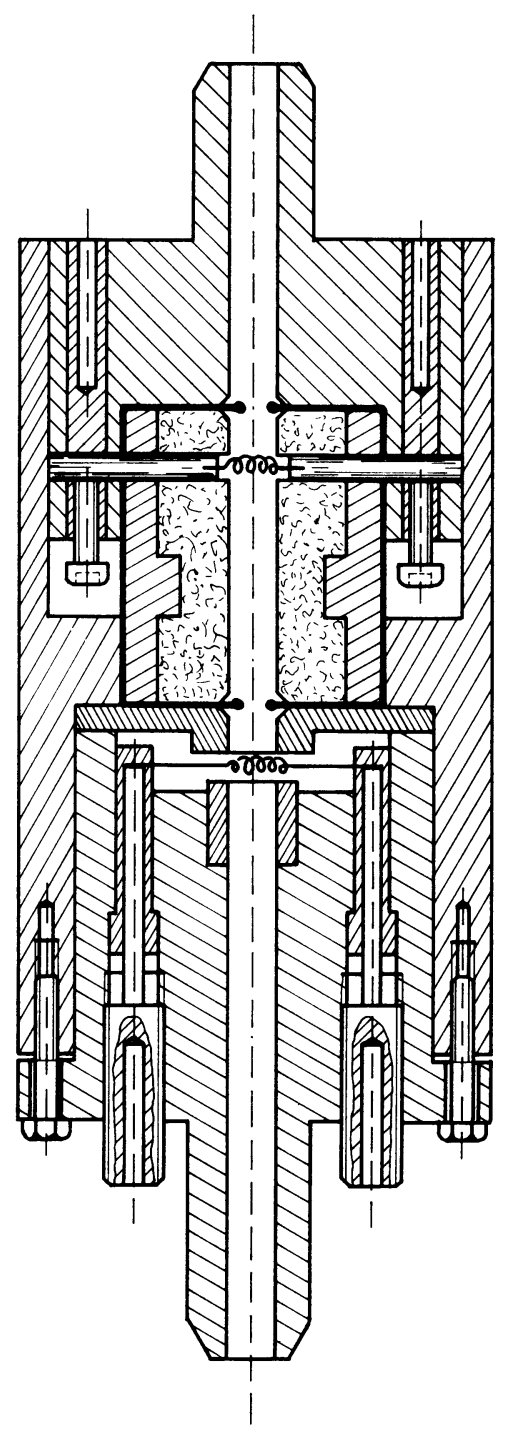

FIG. 2. - Schéma technologique de l'analyseur.

Les filaments de mesure et de référence, constitués par un fil de platine rhodié à $10 \%$ de $0,1 \mathrm{~mm}$ de diamètre, sont alimentés à puissance constante.

Le filament de référence, bobiné sur un mandrin de $0,5 \mathrm{~mm}$, comporte une dizaine de spires.

Le filament de mesure est enroulé en hélice sur un cylindre de section oblongue; cette configuration a l'avantage de couvrir la section de passage du gaz et donc de présenter le maximum de surface en contact avec le courant gazeux, afin que la combustion catalytique soit complète.

En vue de conférer au filament de mesure une activation catalytique rapide, on soumet celui-ci à un chauffage préalable dans de l'air à la température qu'il aura en fonctionnement normal [20].

Notons que les effets d'une variation éventuelle d'activité du filament sont rendus négligeables par la limitation du débit de balayage à une valeur suffisamment faible pour que les gaz résiduels soient exempts d'hydrocarbures [11].

Comme nous l'avons signalé plus haut, la puissance
$Q_{1}$ dissipée dans le filament de mesure est fixée par la température à laquelle il faut porter celui-ci pour qu'il y ait combustion catalytique. Le filament de référence, ne jouant aucun rôle dans la réaction, doit être alimenté à une puissance $Q_{1}^{\prime}$ de telle façon que la thermopile donne une force électromotrice nulle lorsque le doseur est parcouru par de l'air pur. $Q_{1}^{\prime}$ dépend donc de la position relative des soudures des couples thermoélectriques vis-à-vis des filaments. On a tout intérêt à moins chauffer le filament de référence pour augmenter sa durée de vie. Ce résultat peut être obtenu en rapprochant les soudures-aval de la thermopile du filament de référence et en éloignant les soudures-amont du filament de mesure. $Q_{1}^{\prime}$ est alors réglée de façon à annuler la force électromotrice en l'absence de combustion.

Nous avons proposé par ailleurs un modèle mathématique d'un microdébitmètre thermique à une ou deux zones de chauffage [21, 22]. En négligeant les effets du rayonnement des filaments et de la variation des caractéristiques thermiques du fluide et du corps avec la température et en supposant que le 'dégagement de chaleur dû à la réaction exothermique se produit uniquement dans la section de chauffage définie par le premier filament, le modèle proposé dans le second cas s'applique au doseur. Son exploitation permet alors de prévoir l'allure des courbes de répartition de la température du fluide le long de l'axe (Fig. 3). La courbe $a$ représente le fonctionnement du doseur en l'absence de combustion, les quantités de chaleur prises en compte étant $Q_{1}$ pour le filament de mesure et $Q_{1}^{\prime}$ pour le filament de référence.

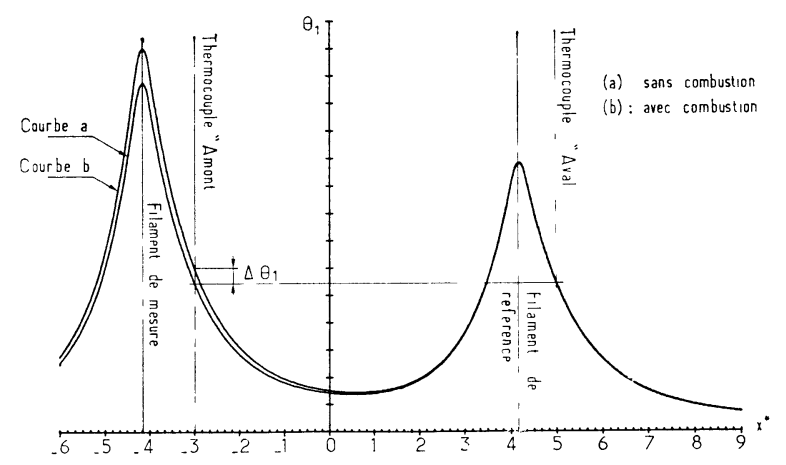

Fig. 3. - Répartition de la température du fluide dans le doseur avec et sans combustion catalytique.

En présence d'hydrocarbure, la réaction se produit et dégage une quantité de chaleur $Q$, fonction de la teneur du mélange, qui s'ajoute à $Q_{1}$, au niveau du premier filament; ceci se traduit par une variation de la répartition suivant l'axe de la température au sein du fluide (Fig. 3, courbe $b$ ). La tension fournie par la thermopile donne une indication de la teneur en hydrocarbure du mélange analysé.

2.2 CholX DU POINT DE FONCTIONNEMENT. - Les différents essais avec de l'air pur ou un mélange air- 
hydrocarbure ont été effectués sur le banc d'étalonnage pneumatique schématisé figure 4 .

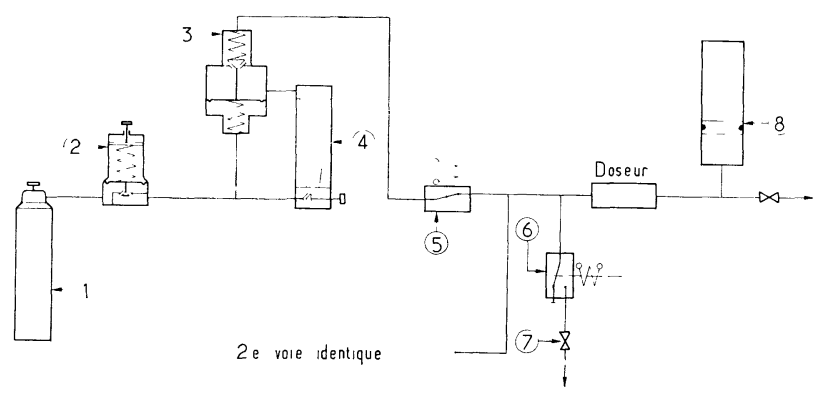

FIG. 4. - Dispositif d'étalonnage.

Après une double détente en sortie de la bouteille haute pression 1, le gaz est maintenu à pression constante par un régulateur de pression 2. Un régulateur de pression différentielle 3 , type montage aval, associé à un débitmètre à flotteur 4 , assure l'affichage, le réglage et la constance du débit vis-à-vis des fluctuations de pression aval. Une logivanne 5 oriente le débit de gaz, soit vers l'extérieur, soit vers le doseur, après passage dans une deuxième logivanne identique 6. Celle-ci est jumelée à une vanne à aiguille 7 et a pour fonction de dévier ou non une partie réglable du débit vers l'extérieur, afin de rendre possible l'application de petits échelons de débit à l'appareil étudié.

A la sortie de l'appareil en essai, le débit de gaz pénètre dans un appareil-étalon 8 du type bulloscope utilisant un piston très léger en PVC à joint de mercure qui se déplace dans un tube calibré cylindrique en verre. On détermine le débit en mesurant le temps nécessaire pour recueillir un volume donné de gaz.

L'appareil-étalon fournissant une mesure du débitvolume, il est nécessaire de travailler à température constante; l'ensemble du circuit décrit ci-dessus a donc été placé dans une enceinte thermostatée à $\pm 0,05^{\circ} \mathrm{C}$.

L'une des voies est alimentée en air pur, l'autre en mélange combustible.

La mise en chauffe s'effectue en balayant l'appareil avec un débit d'air pur. Le régime de fonctionnement atteint, la commande des logivannes 5 permet de réaliser un échelon sur la teneur du gaz en hydrocarbure en commutant les deux voies d'alimentation du doseur.

Un certain nombre de paramètres, tels que : débit du mélange, puissances électriques à dissiper dans les filaments..., sont à fixer préalablement. Notre choix est guidé par l'obligation d'obtenir une combustion complète au niveau du premier filament.

La température du filament de mesure, favorisant la combustion catalytique, dépend de l'hydrocarbure à détecter. Il est donc possible, en réglant la puissance d'alimentation, de procéder à un dosage sélectif d'un seul constituant combustible, si les autres, également contenus dans le mélange, nécessitent des températures plus élevées [16], [23]. Notons que tous nos essais seront conduits avec un mélange air-méthane ; la puissance $Q_{1}$ fournie au filament de mesure est donc choisie en conséquence. La puissance $Q_{1}^{\prime}$, dissipée sur le filament de référence, est alors réglée de façon à annuler la force électromotrice $\Delta e$ délivrée par la thermopile en l'absence de gaz combustible dans le courant gazeux (Fig. 3, courbe a).

Un essai avec un mélange air-méthane à teneur $\varepsilon$ constante s'écoulant à travers le doseur à différents débits (Fig. 5) montre que la sensibilité de l'appareil est maximum pour une valeur optimale du débit d'environ $2,5 \quad 1 . \mathrm{h}^{-1}$.

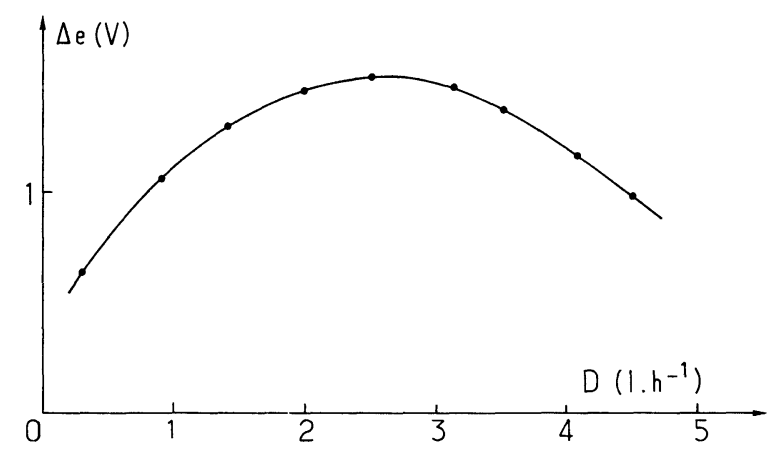

FIG. 5. - Influence du débit sur la sensibilité. $\varepsilon=1 \% \mathrm{CH}_{4}$, $Q_{1}=2,25 \mathrm{~W}, Q_{1}^{\prime}=1,7 \mathrm{~W}$.

Pour des débits inférieurs à cette valeur, la combustion sur le filament de mesure est complète, mais le fluide s'écoulant très lentement se refroidit fortement avant la prise de mesure, d'où la perte de sensibilité constatée. Aux débits supérieurs, par contre, le passage sur le filament de platine est trop rapide et la combustion incomplète.

Des essais systématiques, à débit constant, nous permettent de retrouver des résultats classiques [17] ; en effet, les caractéristiques statiques $\Delta e=f\left(Q_{1}\right)$, tracées pour différentes teneurs en hydrocarbure (Fig. 6), présentent :

- un seuil en deçà duquel la combustion ne peut pas se produire, la température du filament étant trop basse ;

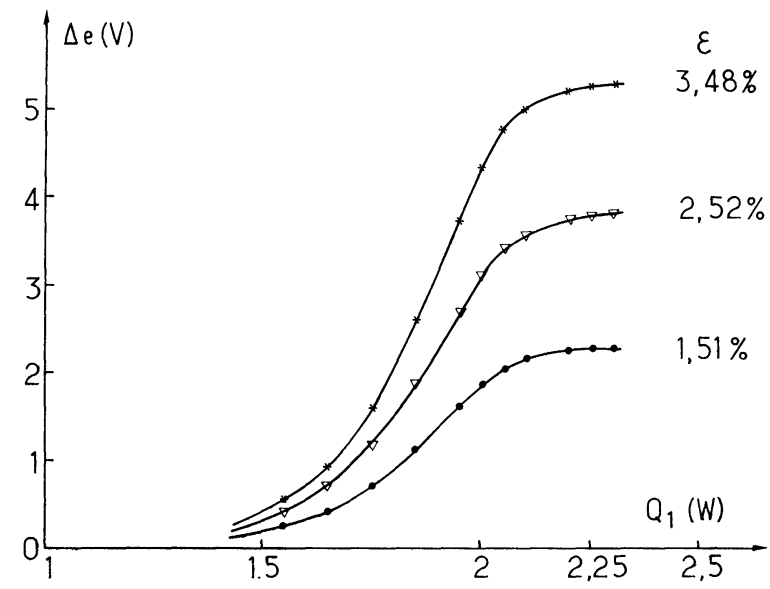

FIG. 6. - Caractéristiques statiques de l'analyseur. 
- au-delà du seuil, une partie quasi linéaire. Dans ce domaine, la réaction devient de plus en plus complète au fur et à mesure que la température du catalyseur s'élève ;

- enfin, un phénomène de saturation correspondant au fait qu'à partir d'une certaine température et avec le débit choisi, la réaction est totale quelle que soit la puissance dissipée. Ce palier se poursuit jusqu'à des puissances conduisant à la rupture du filament.

L'exploitation de ces courbes nous amène à choisir le point de fonctionnement au début du palier de saturation, ce qui conduit à adopter le régime de fonctionnement suivant :

- Débit

$$
D=2,51 \cdot \mathrm{h}^{-1} \equiv 6,9 \times 10^{-7} \mathrm{~m}^{3} \cdot \mathrm{s}^{-1} \text {. }
$$

- Puissance du filament de mesure :

$$
Q_{1}=2,25 \mathrm{~W} \text {. }
$$

- Puissance du filament de référence :

$$
Q_{1}^{\prime}=1,7 \mathrm{~W} \text {. }
$$

2.3 RÉGulation DE PUISSANCE DES FILAMENTS. Pour les variations de puissance envisagées, la figure 3 montre que la distance entre les deux filaments est telle que l'on puisse négliger l'influence d'un filament sur l'autre du point de vue thermique ; aussi, avant d'aborder l'étude dynamique de l'alimentation des filaments de l'analyseur, il convient d'établir d'une façon générale le diagramme fonctionnel représentant le fonctionnement d'un filament chauffé se trouvant dans un courant gazeux.

Il existe une relation entre les variations de la puissance et celles de la tension et de la température du filament; en effet :

$$
Q_{1}+\Delta Q_{1}=\frac{\left(U_{1}+\Delta U_{1}\right)^{2}}{R_{1}(1+\alpha \Delta \theta)}
$$

avec

$$
Q_{1}=\frac{U_{1}^{2}}{R_{1}}
$$

d'où, en négligeant les infiniment petits du second ordre :

$$
\Delta Q_{1}=\frac{2 Q_{1}}{U_{1}} \Delta U_{1}-\alpha Q_{1} \Delta \theta
$$

D'autre part, l'expérience montre que l'on peut valablement considérer que la fonction de transfert liant les variations de la température du fluide à une certaine distance du filament aux variations de la puissance absorbée est du premier ordre :

$$
\frac{\Delta \theta_{1}(p)}{\Delta Q_{1}(p)}=\frac{K_{1}}{1+T_{1} p}
$$

Cette donnée expérimentale, associée à l'éq. (3), permet de tracer le diagramme fonctionnel de la figure 7 et de montrer que le filament constitue un système auto-régulé. Les variations des températures du filament et du fluide sont supposées proportionnelles dans le rapport $\gamma$.

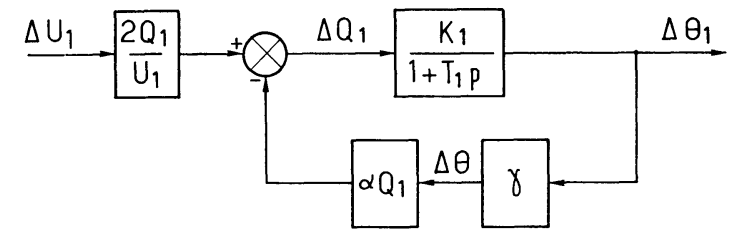

FIG. 7. - Diagramme fonctionnel d'un filament.

Ces hypothèses simplificatrices sont d'autant plus admissibles que les variations de puissance appliquées au filament sont faibles.

De même, l'expérience prouve que la thermopile, constituée de neuf couples thermoélectriques chromelalumel de $0,1 \mathrm{~mm}$ de diamètre, peut être valablement représentée par une fonction de transfert du premier ordre de constante de temps $T_{2}$ et de gain statique $K_{2}$; notons que l'on inclut, dans cette constante, le gain de l'amplificateur de mesure qui est monté en cascade avec la thermopile :

$$
K_{2}=0,189 \mathrm{~V} \cdot{ }^{\circ} \mathrm{C}^{-1} \text {. }
$$

La détermination du gain statique $K_{1}$, des constantes de temps $T_{1}$ et $T_{2}$ et de la constante de proportionnalité $\gamma$ peut se faire à partir de l'essai suivant : on sollicite le filament, placé dans le courant gazeux, en superposant de petits échelons de position $\Delta U_{1}$ à la tension $U_{1}$ d'alimentation et on enregistre simultanément: les variations $\Delta Q_{1}$ de la puissance $Q_{1}$, les variations $\Delta e$ de la force électromotrice délivrée par la thermopile.

Le diagramme fonctionnel représentatif de cet essai et les fonctions de transfert correspondantes sont consignés figure 8 .



FIG. 8. - Diagramme fonctionnel de ı'essai en vue de la détermination de $T_{1}$ et $T_{2}$.

$$
\begin{aligned}
& \int \frac{\Delta Q_{1}(p)}{\Delta U_{1}(p)}=\frac{2 Q_{1}}{U_{1}\left(1+\alpha Q_{1} \gamma K_{1}\right)} \cdot \frac{1+T_{1} p}{1+\frac{T_{1}}{1+\alpha Q_{1} \gamma K_{1}} p} \\
& \int \frac{\Delta e(p)}{\Delta U_{1}(p)}=\frac{2 Q_{1} K_{1} K_{2}}{U_{1}\left(1+\alpha Q_{1} \gamma K_{1}\right)} \cdot \frac{1}{\left(1+\frac{T_{1}}{1+\alpha Q_{1} \gamma K_{1}} p\right)\left(1+T_{2} p\right)} \\
& \int \frac{\Delta Q_{1}(p)}{\Delta U_{1}(p)}=1,8 \cdot \frac{1+0,36 p}{1+0,30 p} \\
& \left\{\frac{\Delta e(p)}{\Delta U_{1}(p)}=\frac{12}{(1+0,30 p)(1+2 p)}\right. \text {. }
\end{aligned}
$$


Le dépouillement de la réponse indicielle $\Delta Q_{1}(t)$ permet de déterminer :

$$
\begin{aligned}
& K_{1}=35,27^{\circ} \mathrm{C} \cdot \mathrm{W}^{-1} \\
& T_{1}=0,360 \mathrm{~s}
\end{aligned}
$$

tandis que l'identification de la réponse indicielle du second ordre $\Delta e(t)$ par la méthode de Hoerner [24] fournit la constante de temps de la thermopile :

$$
T_{2}=2 \mathrm{~s} \text {. }
$$

Notons que cette valeur de la constante de temps correspond à des essais effectués au débit nominal $\left(2,51 \cdot h^{-1}\right)$ choisi au paragraphe précédent.

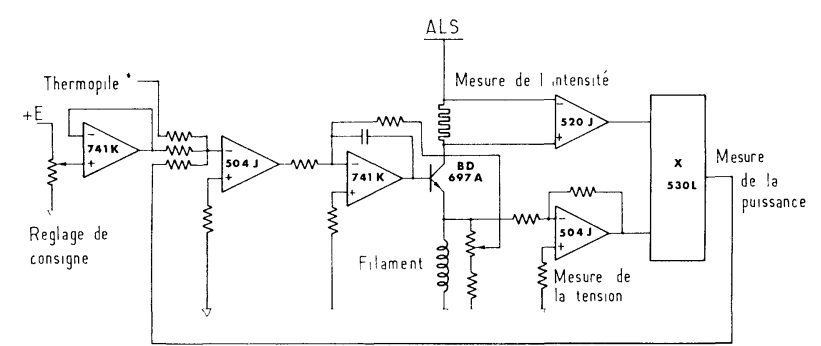

FIG. 9. - Circuit de régulation de puissance d'un filament. Cette entrée n'existe que dans le circuit de régulation du filament de mesure, dans le cas du doseur en méthode de zéro asservi.

Dans le montage adopté pour le doseur, les filaments de mesure et de référence sont alimentés à puissance constante par deux circuits électroniques identiques de régulation de puissance (Fig. 9) qui assurent :

- la mesure séparée de la tension aux bornes du filament et de l'intensité qui le traverse ;

- l'élaboration d'une tension proportionnelle à la puissance dissipée dans le filament ;

- la comparaison de cette tension avec une tension de consigne réglable, d'où l'obtention d'un signal d'erreur qui agit sur l'alimentation du filament.

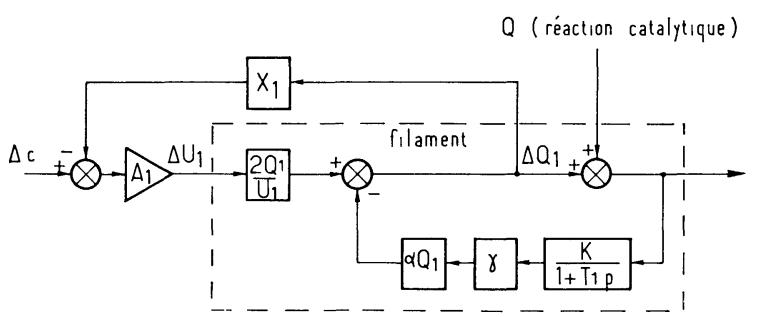

FIG. 10. - Diagramme fonctionnel de la régulation de puissance.

Les diagrammes fonctionnels (Fig. 10) des circuits de régulation de puissance des filaments de mesure et de référence sont analogues à l'entrée $Q$ près qui n'existe que pour le premier filament.

2.4 FONCTION DE TRANSFERT DE L'ANALYSEUR. La quantité de chaleur $Q$ dégagée par la réaction (1) constitue l'entrée de l'analyseur tandis que la sortie



FIG. 11. - Diagramme fonctionnel du doseur.

apparaît sous la forme d'une force électromotrice $\Delta e$ fournie par la thermopile. Il en résulte, après une mise en forme, le diagramme fonctionnel complet de la figure 11 , d'où l'on peut tirer la fonction de transfert du système :

$\frac{\Delta e(p)}{Q(p)}=\frac{K_{1} K_{2}\left(U_{1}+2 Q_{1}\right.}{\alpha Q_{1} \gamma K_{1} U_{1}+U_{1}+2} \frac{A_{1}}{Q_{1} A_{1} X_{1}} \times$

$$
\times \frac{1}{\left(1+\frac{U_{1}+2 Q_{1} A_{1} X_{1}}{\alpha Q_{1} \gamma K_{1} U_{1}+U_{1}+2 Q_{1} A_{1} X_{1}} T_{1} p\right)\left(1+T_{2} p\right)}
$$

soit :

$$
\frac{\Delta e(p)}{Q(p)}=\frac{6,66}{(1+0,36 p)(1+2 p)}
$$

Le gain statique $\Delta e / Q$ est de $6,66 \mathrm{~V} . \mathrm{W}^{-1}$. En se ramenant à la quantité de chaleur dégagée par la réaction d'un mélange à $1 \%$ de méthane, dans les conditions d'essai fixées au paragraphe 2.2 , on obtient une sensibilité de $1,525 \mathrm{~V}$ par \% de méthane. La fonction de transfert (5) présente deux constantes de temps : l'une due au filament de mesure, l'autre à la thermopile. Le temps de réponse à $5 \%$, calculé à partir de la relation (6), est théoriquement de $8 \mathrm{~s}$ [25].

La vérification expérimentale de ce résultat nécessite l'envoi sur le doseur d'un échelon de concentration de méthane. Le gaz se déplaçant à vitesse constante, le temps nécessaire au parcours de la distance organe de commutation-premier filament fait apparaître un retard pur dont il serait aisé de tenir compte pour la détermination du temps de réponse de l'appareil. En fait, les phénomènes de diffusion excluent la possibilité d'obtenir un tel signal.

Dans le cas de l'utilisation de la rampe d'étalonnage de la figure 4, nous avons cherché à déterminer l'allure de l'entrée réellement appliquée au système. Celle-ci est représentée figure 12 en supposant que les deux gaz en présence diffusent suivant la deuxième loi de Fick [26] et en éliminant les déformations du plan de séparation air pur-mélange dues au fonctionnement des logivannes 5 et aux accidents de tuyauterie. 


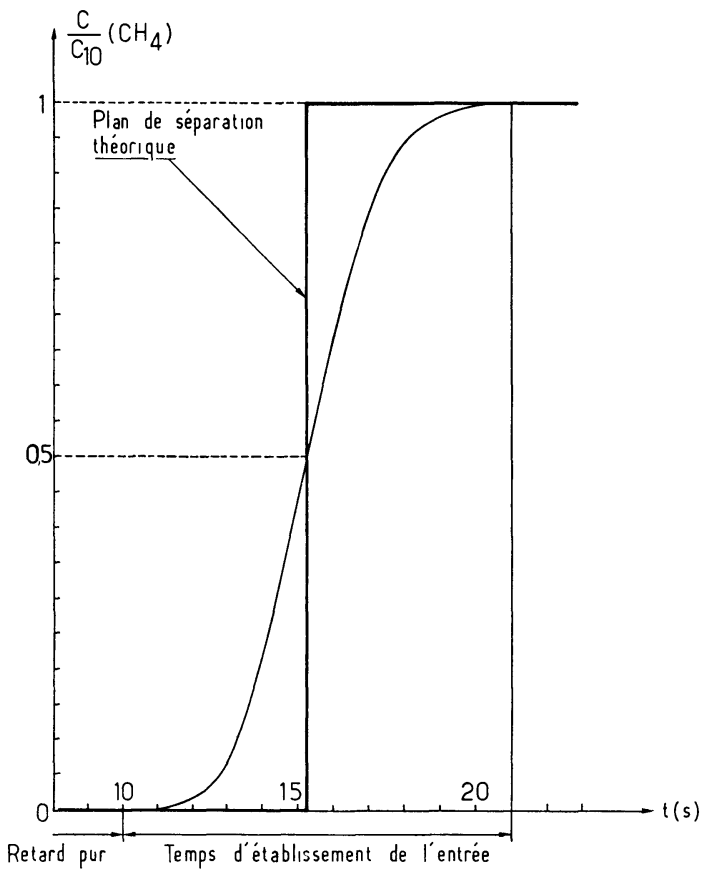

Fig. 12. - Allure de la concentration en $\mathrm{CH}_{4}$ du mélange, au niveau du filament de mesure.

L'évolution, du fait de la diffusion, de la forme de la surface de séparation au cours du temps, entraine, au niveau du premier filament, une diminution du retard pur et un établissement progressif de la concentration en méthane du mélange (cf. annexe 2).

Dans ces conditions, il est possible d'évaluer le temps $T^{*}$ mis par le signal de sortie du doseur pour atteindre $95 \%$ de sa valeur finale. On peut l'obtenir en calculant la réponse $\Delta e(t)$ de l'appareil à l'entrée, définie ci-dessus, par la méthode de la transformée en $z$ utilisée dans l'étude des systèmes échantillonnés [27]. Le temps $T^{*}$ ainsi calculé est de $28 \mathrm{~s}$; or, le temps mesuré sur l'enregistrement de la réponse est égal à $86 \mathrm{~s}$.

Notons que, dans ces deux dernières valeurs, est inclus le temps de retard de dix secondes, non imputable à l'appareil lui-même.

Remarquons que le temps $T^{*}$, calculé théoriquement, doit être en fait majoré pour tenir compte des hypothèses simplificatrices émises lors de la détermination de l'entrée : mode de commutation des vannes, canalisations présentant des coudes et des changements de section, ...

Cependant, l'expérience montre que la présence, dans la fonction de transfert (6) du doseur, de deux constantes de temps ne suffit pas à rendre compte du régime transitoire dans son intégralité.

Outre la cinétique de l'établissement permanent de la réaction exothermique (1) qui est très difficile à préciser, on peut noter que le dégagement de chaleur dû à cette combustion donne lieu à un nouvel état d'équilibre de l'ensemble. Cette mise en équilibre thermique du corps, qui a été négligée lors de l'étude du filament, entraîne en plus une évolution lente et de faible amplitude $(7 \%$ du régime permanent) du signal de sortie qui se traduit par un allongement important du temps $T^{*}$ mesuré expérimentalement par rapport à sa valeur théorique.

Compte tenu des remarques précédentes, on peut considérer que le modèle dynamique proposé rend compte valablement du comportement du doseur à l'exception de la dérive due à l'établissement d'un nouveau régime thermique.

3. Analyseur fonctionnant en méthode de zéro asservi. - 3.1 PRINCIPE DE LA MESURE. - Dans l'analyseur étudié précédemment, on fournit au filament de mesure une puissance électrique $Q_{1}$ constante. Lors de la combustion, la puissance s'élève à : $Q_{1}+Q$, d'où l'évolution vers un nouvel état d'équilibre thermique de l'ensemble, ce qui se traduit par l'augmentation du temps de réponse, signalé plus haut. Pour éliminer cet inconvénient, il est nécessaire de maintenir inchangé cet état d'équilibre, qu'il y ait réaction ou non. On peut parvenir à ce résultat en agissant sur la puissance électrique délivrée au filament de mesure, afin de conserver constante la quantité : $Q_{1}+Q$.

Dans la mesure où le dégagement de chaleur $Q$ dû à la combustion est localisé dans la zone de chauffage, le maintien des quantités : $Q_{1}+Q$ et $Q_{1}^{\prime}$ à des valeurs fixes entraîne une répartition unique (courbe $a$, Fig. 3) des températures suivant l'axe, quel que soit le régime de fonctionnement. Or, la force électromotrice $\Delta e$ obtenue aux bornes de la thermopile est due à la différence de températures existant entre deux points fixes de l'axe ; elle constitue un signal permettant de s'assurer de la constance de la quantité $: Q_{1}+Q$.

Le réglage initial choisi auparavant $(\S 2.2)$ est tel que $\Delta e$ est nulle en l'absence de combustion. L'apparition d'une valeur non nulle de $\Delta e$ est liée à l'existence de $Q ; \Delta e$ peut alors servir à commander la puissance électrique.

En conclusion, la variation $\Delta Q_{1}$ de la puissance électrique constitue une mesure de $Q$, donc de la teneur en hydrocarbure du mélange.

\subsection{Etude STATiQue et DYNAMiQue De L'ANALYSeUr.} - Le diagramme fonctionnel de la figure 13 représente l'asservissement réalisé.

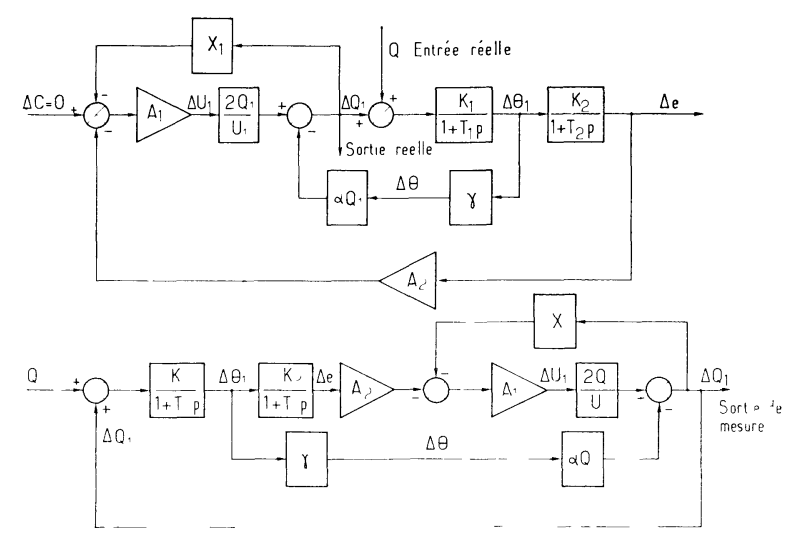

Fig. 13. - Diagramme fonctionnel du doseur en méthode de zéro asservi. 
On peut en déduire les fonctions de transfert suivantes :

$$
\left\{\begin{array}{c}
\frac{\Delta Q_{1}(p)}{Q(p)}=-G_{1} \frac{1+\tau p}{1+A p+B p^{2}} \\
\frac{\Delta e(p)}{Q(p)}=G_{2} \frac{1}{1+A p+B p^{2}}
\end{array}\right.
$$

avec

$$
\begin{aligned}
& \begin{aligned}
G_{1} & =\frac{K_{1} Q_{1}\left(\alpha \gamma U_{1}+2 A_{1}\right.}{N} \underline{\left.A_{2} K_{2}\right)} \\
G_{2} & =\frac{K_{1} K_{2}\left(U_{1}+2 A_{1} Q_{1} X_{1}\right)}{N} \\
\tau & =\frac{\alpha \gamma U_{1} T_{2}}{\alpha \gamma U_{1}+2 A_{1} A_{2} K_{2}}
\end{aligned} \\
& \left\{=\frac{\alpha Q_{1} \gamma U_{1} T_{2}+\left(U_{1}+2 A_{1} Q_{1} X_{1}\right)\left(T_{1}+T_{2}\right)}{N}\right. \\
& B=\frac{\left(U_{1}+2 A_{1} Q_{1} X_{1}\right) T_{1} T_{2}}{N}
\end{aligned}
$$

en posant :

$$
\begin{array}{r}
N=U_{1}+2 A_{1} Q_{1} X_{1}+2 A_{1} A_{2} Q_{1} K_{1} K_{2}+ \\
+\alpha Q_{1} \gamma K_{1} U_{1}
\end{array}
$$

soit numériquement :

$$
\left\{\begin{aligned}
\frac{\Delta Q_{1}(p)}{Q(p)} & =-0,93 \frac{1+0,17 \times 10^{-3} p}{1+0,165 p+0,05 p^{2}} \\
\frac{\Delta e(p)}{Q(p)} & =0,465 \frac{1}{1+0,165 p+0,05 p^{2}} .
\end{aligned}\right.
$$

Du fait de la structure du diagramme fonctionnel de l'analyseur, le gain statique $\Delta Q_{1} / Q$ est égal à 0,93 , soit $213 \mathrm{~mW}$ pour $1 \%$ de méthane brûlé. Les caractéristiques statiques de la figure 14 ont la même

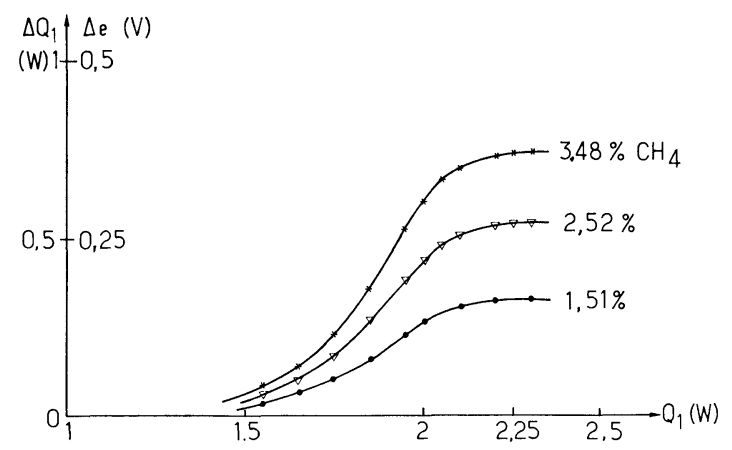

FIG. 14. - Caractéristiques statiques du doseur asservi.

allure que celles du doseur non asservi. La courbe d'étalonnage du doseur (Fig. 15), pour le point de fonctionnement choisi, peut être déduite de ces caractéristiques.

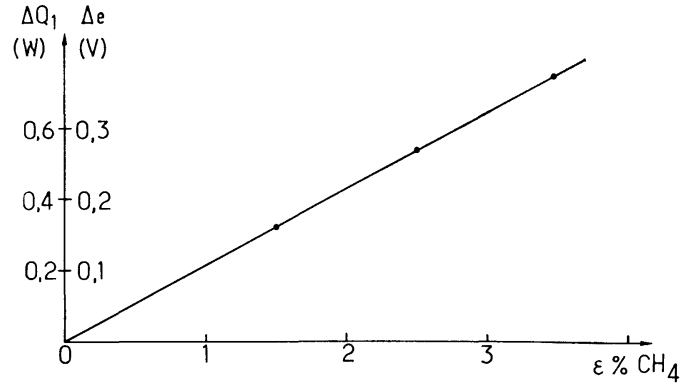

FIG. 15. - Courbe d'étalonnage du doseur asservi.

Le temps de réponse théorique à $5 \%$ est de $3 \mathrm{~s}$. Compte tenu de l'entrée réellement appliquée au système (cf. \$ 2.4), le calcul par la méthode de la transformée en $z$ [27] donne un temps $T^{*}$ théorique de $23 \mathrm{~s}$, y compris le retard pur de $10 \mathrm{~s}$. Le temps déterminé expérimentalement est de $33 \mathrm{~s}$. La différence entre ces deux valeurs s'explique, d'une part, par les hypothèses simplificatrices admises lors du calcul de la forme de l'entrée et, d'autre part, par la cinétique de l'établissement de la réaction.

La diminution sensible du temps de réponse entre les deux versions de l'analyseur confirme la disparition des changements de régime thermique du corps $d u$ doseur.

Toutefois, l'absence d'intégration, pour des raisons de stabilité, dans la fonction de transfert en boucle ouverte de l'analyseur entraîne la présence d'une erreur permanente et, par voie de conséquence, l'existence de très faibles changements de régime thermique. Le calcul montre que, pour une variation de teneur en méthane correspondant à la totalité de l'échelle de l'appareil (soit $5 \%$ ), l'écart de températures détecté par la thermopile est au maximum de $2,8^{\circ} \mathrm{C}$. On peut donc valablement considérer que la température du corps de l'analyseur reste constante, ce qui est en accord avec l'expérience.

Il est aisé de remédier à cet inconvénient en introduisant un correcteur du type proportionnel-intégral, au niveau de l'amplificateur $A_{2}$ de la figure 13 , de fonction de transfert :

$$
C(p)=\frac{1+T_{2} p}{T_{2} p} .
$$

Le correcteur choisi élimine ainsi l'influence de la constante de temps de la thermopile et annule l'erreur statique constatée ci-dessus.

4. Conclusions. - L'étude expérimentale du doseur montre que le modèle d'un microdébitmètre thermique à deux zones de chauffage, que nous avons proposé par ailleurs [22], rend compte qualitativement de son fonctionnement et permet sa détermination approchée.

En ce qui concerne les performances dynamiques de l'appareil, les diagrammes fonctionnels établis et les fonctions de transfert qui en découlent sont en bon accord avec l'expérience ; leur exploitation a conduit à 
l'élaboration d'un doseur fonctionnant en méthode de zéro asservi.

Cette version du doseur à combustion catalytique apporte des solutions convenables aux défauts évoqués pour les appareils existants ; en particulier, elle permet :

- d'éliminer la dérive de zéro ;

- d'accroître considérablement la durée de vie du filament en le maintenant à température constante avec ou sans combustion ;

- d'obtenir un temps de réponse optimum.

Le doseur présenté nous semble particulièrement bien adapté au dosage en continu des hydrocarbures gazeux.

\section{ANNEXE 1}

\section{Notations.}

pourcentage d'hydrocarbure du mélange ; quantité de chaleur par unité de temps apportée au fluide par combustion catalytique au niveau du filament de mesure, dans la section de chauffage (W) ;

$Q_{1}, U_{1}, R_{1}$ quantité de chaleur par unité de temps (W), tension d'alimentation $(\mathrm{V})$, résistance $(\Omega)$ relatives au filament de mesure ;

$\Delta Q_{1}, \Delta U_{1}$ petites variations des grandeurs précédentes ;

$Q_{1}^{\prime} \quad$ quantité de chaleur par unité de temps (W) relative au filament de référence ;

$R_{1} \quad$ rayon intérieur du doseur (m);

$x \quad$ abscisse de la section droite considérée (m) ;

$x^{*} \quad$ abscisse réduite $x^{*}=x / R_{1}$;

$U \quad$ vitesse moyenne du fluide dans le tube $\left(\mathrm{m} . \mathrm{s}^{-1}\right)$;

$D \quad$ débit volumétrique du fluide $\left(\mathrm{m}^{3} \cdot \mathrm{s}^{-1}\right)$;

$\theta_{1} \quad$ température moyenne du fluide par rapport à la température ambiante $\left({ }^{\circ} \mathrm{C}\right)$;

$\Delta \theta, \Delta \theta_{1} \quad$ variation de température du filament de mesure, variation de température du fluide à une distance donnée du filament $\left({ }^{\circ} \mathrm{C}\right)$;

$\gamma=\frac{\Delta \theta}{\Delta \theta_{1}}$

rapport de proportionnalité entre les variations de température ;

$\alpha$ coefficient de variation de la résistivité du platine en fonction de la température ;

$K_{1} \quad$ gain statique liant les variations de température $\Delta \theta_{1}$ aux variations de puissance $\Delta Q_{1}\left({ }^{\circ} \mathrm{C} . \mathrm{W}^{-1}\right)$;

$T_{1} \quad$ constante de temps du filament de mesure $(s)$;
$K_{2}$

$T_{2}$

$A_{1}$

$X_{1}$

$p$ gain statique liant la $\mathrm{f}$. é. $\mathrm{m}$. délivrée par par la thermopile $\Delta e$ à la variation de température du fluide $\Delta \theta_{1}\left(\mathrm{~V} .{ }^{\circ} \mathrm{C}^{-1}\right)$; constante de temps de la thermopile (s); gain de l'amplificateur ; gain du multiplieur ; opérateur de Laplace.

\section{ANNEXE 2}

Détermination de l'entrée de l'analyseur. - La diffusion du méthane dans l'air se fait suivant la deuxième loi de Fick [26], [28] :

$$
\frac{\partial c}{\partial t}=\frac{\partial}{\partial x}\left(D \frac{\partial c}{\partial x}\right)
$$

La résolution de cette équation, en supposant que le coefficient de diffusion $D$ est constant, permet de déterminer la concentration du produit à une distance $x$ du plan de séparation initial et pour un temps $t$ donné.

Soient $c_{10}$ et $c_{20}$ les concentrations initiales en méthane de part et d'autre du plan de séparation (air pur-mélange); la concentration $c(x, t)$ a pour expression :

$$
c=\frac{c_{10}+c_{20}}{2} \pm\left(c_{10}-c_{20}\right) G(X)
$$

avec

$$
\left\{\begin{aligned}
G(X) & =\frac{1}{\sqrt{2 \pi}} \int_{0}^{X} \mathrm{e}^{-u^{2} / 2} \mathrm{~d} u \\
X & =\frac{x}{\sqrt{2 D t}} .
\end{aligned}\right.
$$

Dans le cas du méthane: $D=0,163 \mathrm{~cm}^{2} \cdot \mathrm{s}^{-1}$ [29].

Soit $L_{0}$ la distance : logivanne-filament de mesure. Le plan de séparation se déplaçant à la vitesse moyenne $U$ du fluide, au temps $t$, la distance $x$ du plan de séparation au filament de mesure est :

$$
x=L_{0}-U t .
$$

Pour chaque instant $t$, on peut donc: déterminer la quantité $X$ par la relation (A.2.4), déduire la valeur de la fonction de Galton $G(X)$ à l'aide de tables numériques [30] et ainsi calculer la concentration $c(t)$ au niveau du premier filament.

La figure 12 montre, pour notre installation d'essai, l'évolution de la concentration en méthane du gaz en contact avec le filament de mesure en fonction du temps.

\section{Bibliographie}

[1] Monomakhoff, A., Rev. Ind. Min. 38 (1956) 228-242.

[2] Monicard, R., Rev. Inst. Fr. Pet. VIII (1953) 555-560.

[3] Denis, J., Parc, G., Rev. Inst. Fr. Pet. 17 (1962) 14731501.
[4] Susumu Namba, Rev. Sci. Instrum. 30 (1959) 642-645.

[5] Riken Keiky, Veb Carl Zeiss Jena (Notices).

[6] Nash, H., Hall, J. R., Allgood, H. Y., Burnett, J. A., Ann. Chem. 24 (1952) 1650-1652. 
[7] Leeds and Northrup, Gas Analysis systems (LC 5, UA 5), Hartmann and Braun (Caldos 2), Geoservices (G. D. 1) (Notices).

[8] Beckman (215A, 315A, 415A), Schlumberger (ANIR 11), Jasco (IRA 1, IRA 2), Cosma... (Notices).

[9] Devore, P., Thèse Lyon 1959.

[10] Winter, K., Rev. Ind. Min. 37 (1955) 43-49.

[11] Domanski, B., Jourdan, A., Eyraud, Ch., Chim. Anal. 38 (1956) 322-327.

[12] Belugou, P., de Vergeron, M., Monomakhoff, A., Rev. Ind. Min. 46 (1964) 57-63.

[13] Monomakhoff, A., Rev. Ind. Min. 50 (1968) 21-40.

[14] Compagnie Auxiliaire des Mines Douai (Verneuil 54, Verneuil miniature...) (Notices).

[15] Eyraud, Ch., Perrin, M., Chim. Anal. 33 (1951) 132-140.

[16] Eyraud, Ch., Domanski, B., Devore, P., Botazzi, H., Chim. Anal. 43 (1961) 136-146.

[17] Jutard, A., Thèse Lyon 1964.

[18] Eyraud, Ch., Jutard, A., Richard, M., Eyraud, L., Brevet français $\mathrm{n}^{\circ}$ 1.407.736, (1964).
[19] Bussière, P., Domanski, B., Eyraud, Ch., Prettre, M., C. R. Hebd. Séan. Acad. Sci. 243 (1956) 1870-1872.

[20] Domanski, B., Thèse Lyon 1957.

[21] Jutard, A., Scavarda, S., Betemps, M., Automatisme XIX (1974) 42-49.

[22] Jutard, A., Scavarda, S., Betemps, M., KollmansberGER, M., J. Phys. E : Sci. Instrum. 8 (1975) 209-216.

[23] Monicard, R., Rev. Inst. Fr. Pet. VI (1951) 379-383.

[24] Hoerner, G. M., Cont. Ing. 9 (1962) 93-95.

[25] Gille, J. C., Decaulne, P., Pelegrin, M., Dynamique de la commande linéaire (Dunod) 1967 ch. 8, 234-236.

[26] Jost, W., Diffusion in solids, liquids, gases (Academic Press) 1960, ch. 1 et 10.

[27] SeVely, Y., Systèmes et asservissements linéaires échantillonnés (Dunod) 1969, ch. 3, 44-45.

[28] Adda, Y., Philibert, J., La diffusion dans les solides (Presses Universitaires de France) 1966.

[29] Hirschfelder, J. O., Curtiss, Ch. F., Bird, R. B., Molecular theory of gases and liquids (John Wiley) 1954, 579.

[30] Boll, M., Tables numériques universelles (Dunod) 1964. $3^{\text {e }}$ éd. 